\title{
The Role of Global Observations for Climate and Other Applications
}

May 2005

T.P. Ackerman

Pacific Northwest National Laboratory

Work supported by the U.S. Department of Energy, Office of Science, Office of Biological and Environmental Research 


\section{Disclaimer}

This report was prepared as an account of work sponsored by the U.S. Government. Neither the United States nor an agency thereof, nor any of their employees, makes any warranty, express or implied, or assumes any legal liability or responsibility for the accuracy, completeness, or usefulness of any information, apparatus, product, or process disclosed, or represents that its use would not infringe privately owned rights. Reference herein to any specific commercial product, process, or service by trade name, trademark, manufacturer, or otherwise, does not necessarily constitute or imply its endorsement, recommendation, or favoring by the U.S. Government or any agency thereof. The views and opinions of authors expressed herein do not necessarily state or reflect those of the U.S. Government or any agency thereof. 


\section{Acronyms}

ARM Atmospheric Radiation Measurement (Program)

BSRN Baseline Surface Radiation Network

NASA National Aeronautics and Space Administration

NOAA National Oceanic and Atmospheric Administration

NPOES NOAA Polar-Orbiting Operational Environmental Satellites 


\section{Contents}

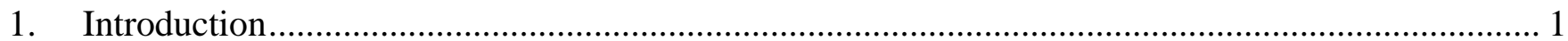

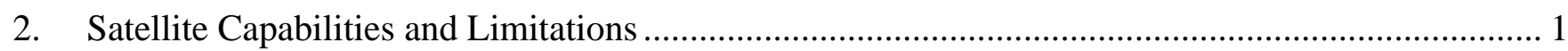

3. Satellite Plus Surface-Based Measurements: A Three-Tiered Strategy ........................................ 2

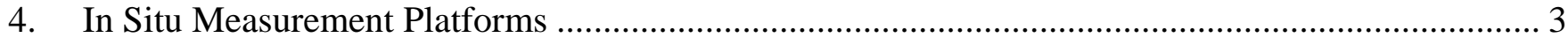

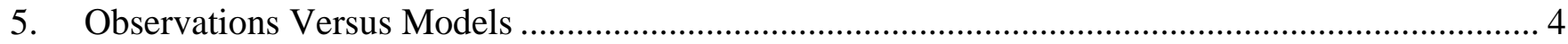

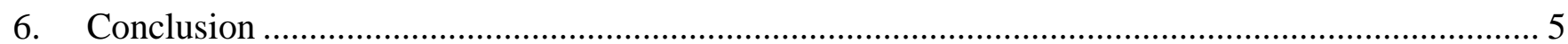




\section{Introduction}

Most of the current earth-observing systems have been designed primarily for the needs of weather forecasting. Weather forecasting is an initial condition problem; the success of the forecast is heavily dependent on the quality of the specified initial state of the atmosphere. Thus, weather forecasting observing systems tend to focus on determining the 3D values of the state variables of the system - namely temperature, humidity, and wind vector. While weather forecasting requires accurate observations, spatial patterns and relative accuracy across those patterns are the primary concern.

Climate, on the other hand, is a boundary condition problem, i.e., climate simulation depends on knowing the energy fluxes into and out of the system, and quantities such as $\mathrm{CO}_{2}$ that affect the flow of those energy fluxes in the system. Consequently, climate-observing systems must extend beyond measurements of state variables to flux measurements of radiation energy and water. We focus on these two cycles because the dominant forms of energy transfer in the climate system (solar energy, thermal infrared energy, evaporation, and condensation) involve these two quantities. Further, because climate is a search for small system trends and imbalances in the midst of large weather variability, climate observations require a much higher degree of precision than do weather observations.

\section{Satellite Capabilities and Limitations}

Satellite instruments, not surprisingly, provide the backbone of both weather and climateobserving systems. Satellites provide wide area coverage by their orbital characteristics and the use of cross-track scanning instruments. In the past several decades, we have dramatically increased the number of satellites and instruments, their measurement resolution and variety, and improved their calibration. This trend has benefited both weather forecasting and climate. We now routinely measure temperature profiles, water vapor path amount and some profiles, cloud occurrence and other properties, wind direction and speed by tracking cloud movements, aerosol column amount, and a wide variety of other parameters. There are, however, some distinct limitations to satellites that should be noted, especially with regard to climate.

1. Satellites provide relatively poor temporal resolution, which is a particular problem for the measurement of rapidly changing fields such as clouds, because of their infrequent orbital overpasses (typically twice a day).

2. Satellite measurements are predominantly made using radiometers, which are passive instruments. Radiometers measure the radiance exiting the top of the atmosphere along a specific direction. The exiting radiation, whether scattered solar or emitted thermal radiation, is affected by the atmospheric composition and the surface characteristics along the line of sight. In mathematical terms, it is the result of an integral, or summation, process. Our task as scientists is to unscramble the summation to identify the component parts of the sum. As any mathematician knows, this is a difficult and often ill-posed problem. More recently, we have begun to explore the use of active sensors in space, such 
as radars and lidars. While these systems hold great promise, they are limited by size and weight considerations, potentially short lifetime in space, and inability to scan large crosstrack paths.

3. Satellite measurements are ill-suited to measure fluxes of energy at the earth surface or within the atmosphere because of the remote nature of the observations. We arrive at satellite estimates of surface and atmosphere fluxes by extrapolation and estimation of measurements of state variables.

4. Satellite measurements are often inadequate to explore detailed climate processes because measurements of important quantities are not taken simultaneously in time and space.

Thus, the cross-correlation terms, which are extremely important in determining causation, are not measured. The National Aeronautics and Space Administration (NASA) A-train concept, which includes some five or six satellites flying in close formation, is an attempt to address this problem, but will only do so for a limited time determined by the lifetime of individual satellites and instruments.

The limitations of satellite measurements provide an important argument for augmenting the satellite-observing system with ground-based observing systems for climate in particular, but also for other applications such as weather forecasting, environmental prediction systems, and research. Ground-based systems have the advantage of providing high temporal resolution measurements, usually on the order of seconds. These measurements, when acquired continuously, provide a complementary look at the atmosphere compared to the broad spatial resolution of satellite instruments. It is technically possible, although expensive, to acquire simultaneous measurements of many different quantities in the atmosphere and at the surface, including both state variables and energy fluxes. The combination of active and passive sensor measurements to retrieve atmospheric properties is now a well-established technique that allows us to investigate climate and weather processes in unprecedented detail. In addition, these ground-based measurements provide the best way to evaluate the accuracy and representative nature of satellite measurements.

\section{Satellite Plus Surface-Based Measurements: A Three-Tiered Strategy}

The climate-observing system of the future must be a combination of satellite and groundbased systems. Considerable thought has been given to the satellite component of the observing system. Plans for National Oceanic and Atmospheric Administration (NOAA) Polar-Orbiting Operational Environmental Satellites (NPOES) and geostationary payloads are well underway. The ground-based component, however, has received much less attention. This is due to a number of reasons. One major reason is the sheer complexity of ground-based systems and possible networks that have confused the discussion; another is the rapidly escalating cost of ground-based systems as their complexity and extent grows. We propose here a three-tiered system that has the advantages of relative simplicity and controlled cost.

- $\quad 1^{\text {st }}$ level: a broad network of basic surface flux measurements such as those currently being carried out by the Baseline Surface Radiation Network (BSRN). The BSRN is largely a volunteer organization of scientists making surface radiation and meteorological 
measurements. The BSRN establishes protocols and procedures that ensure accurate measurements and available data. The network provides invaluable monitoring of trends and evaluation of satellite extrapolations of surface energy fluxes. This network should be extended, particularly to underrepresented areas of the globe, to encompass hundreds of sites around the world.

- $\quad 2^{\text {nd }}$ level: intermediate sites that incorporate highly reliable instruments to measure water vapor, low cloud base heights, and cloud occurrence along with the BSRN-type measurements. Such instruments are readily available now and could be improved if the manufacturing companies knew that a steady market existed for their products. These sites, similar to but expanded slightly from the U.S. Weather Service Automated Weather Station sites, would be highly useful for both weather forecasting needs and climate studies. The required number of such sites is probably on the order of a hundred, distributed across climatic regimes of interest.

- $\quad 3^{\text {rd }}$ level: high-end remote sensing sites that incorporate the very best ground-based technology. The Atmospheric Radiation Measurement (ARM) Program sites are currently the best example of this class, but there are another four or five such sites being run by other countries, predominantly in Europe. These sites seek to measure all climatically important properties of the atmosphere at high resolution, temporally and vertically. The datasets are critically important for understanding climate processes, satellite validation, and model comparison and validation. While an argument can be made for an extensive network of such sites, a small network of perhaps 15 to 20 such sites is probably adequate.

This three-tiered structure is critically important. The $1^{\text {st }}$ level supplies the broad-based global monitoring required by climate, but measurements at these sites are informed and interpreted by the measurements made at the high-end sites. The intermediate sites provide a way to scale the measurements of the high-end sites to larger regions and provide a broader network of measurements for satellite and model validation.

\section{In Situ Measurement Platforms}

There is a third component of observations that is more difficult to fit into the discussion of a global-observing system, but is nonetheless critical. Both satellite and ground-based systems retrieve atmospheric properties by remote sensing using measured radiative signals to infer atmospheric composition and properties. The ultimate validation of these retrieval techniques is by in situ observations from airborne platforms. The research aircraft capability in this country and around the world is currently in a downward spiral and shows little signs of recovery. It is vitally important that any discussion of a long-term climate observing system include serious consideration of support for aircraft platforms, both manned and unmanned, and for instrument development for these platforms. As noted earlier, climate observations require very high precision, which in turn demands continuous validation. 


\section{Observations Versus Models}

Finally, we need to consider the relationship between climate observations and climatemodeling capability. This is an extraordinarily complicated subject and the discussion here can only touch upon some of the important issues. The intersection between observations and modeling occurs along several different fronts, principally,

1. process understanding

2. parameterization development and testing

3. model evaluation.

To incorporate essential atmospheric physics and chemistry into models, we need to understand and model it at the very highest level of detail. Research at this level is a complex web of theory and empiricism that requires accurate, high-resolution data. Ground-based sites, such as the ARM sites, have proved to be vital elements in this process because they supply this class of data. Special experiment periods that bring aircraft to these sites are especially valuable.

Once the detailed physics and chemistry are known, the next step is to extract the core relationships to be included into climate models as sub-grid scale parameterizations. This again is a complex task. Ground-based and satellite systems provide the broad base of global and temporal observations against which such parameterizations need to be developed and tested. For example, one might develop a parameterization based on data acquired at mid-latitude sites in the United States and Europe. Before implementing such a parameterization in global models, it needs to be tested against tropical and polar data to be sure that it provides an adequate representation of atmospheric processes in these very different climatic regimes.

As parameterizations are incorporated into climate models, there is an ongoing need to test model results against observations. The question here is not one of weather forecasting, i.e., did we get the right weather on a given day, but one of climatology, i.e., did we get the correct distribution of state parameters and energy fluxes over a decade-long simulation? Both satellite and ground-based datasets are critical elements of such evaluations. To date, we have largely focused on top-of-atmosphere measurements. These measurements do not constrain cloud properties adequately, leading to much of the disagreement currently seen in simulations of climate change due to increasing greenhouse gas concentrations. Ground-based system offer unique datasets that can and are being used to understand cloud processes and cloud feedbacks in global models.

While we have presented these three steps as a linear progression, they should really be considered as an ongoing, intertwined ribbon. As we diagnose model problems, we then turn back to our understanding of fundamental physics to find out what is incorrect. This in turns leads us to formulate new parameterizations and then to test these against available data. 


\section{Conclusion}

As we look to the future of climate change, climate research, and climate modeling, there is no doubt that progress in this arena will be intimately connected to data availability. Climateobserving systems must include a balanced approach based on a combination of satellite and ground-based systems. Neither is adequate by itself. In situ (aircraft) observations must also be included as a critical component for evaluation and assessment of accuracy. Without an integrated observing system, it is extremely unlikely that we will be able to develop or validate the climate models required to understand the problem of climate change we face. 